\title{
High throughput root phenotyping for cereal plants using spatial distribution in polar coordinate system
}

\author{
Jinhai Cai $^{\text {a }}$, Van Lam Nguyen ${ }^{\text {b }}$, Matthew Wheal ${ }^{\text {, }}$, James Stangoulis ${ }^{\text {b }}$, Stan Miklavcic ${ }^{\text {a }}$ \\ ${ }^{a}$ Phenomics and Bioinformatics Research Centre (PBRC), University of South Australia, Mawson Lakes SA \\ 5095, Australia \\ ${ }^{b}$ School of Biological Sciences, Flinders University of South Australia, GPO Box 2100, South Australia \\ 5001, Australia \\ Email: Jinhai.Cai@unisa.edu.au
}

\begin{abstract}
With the growth of the global population, the demand for better living standards and the accelerating use of grain for biofuel production, the pressures on global grain supplies are becoming immensely high. To satisfy the growing, worldwide demand for grain, it is necessary to improve the productivity of existing farmland. This is a very challenging task in the face of global environmental change. Plant breeders need to focus on traits with the greatest potential to increase yield. In this paper, we focus on the phenotyping of cereal plant roots as roots are the hidden parts of plants and are the principal organ of plants for the absorption of water and the uptake of nutrients from soil. It is well known that the root system architecture is determined by environmental factors, particularly soil conditions as well as plant's genetic makeup. This is typically called the genotype by environment (GxE) interaction. Clearly, the phenotype of a genotype is environmentally dependent. Various studies have been conducted on the effects of abiotic stresses on plant growth and adaptation, including the phenotype-genotype mapping, which has been applied to the problems from finding genes association to plant breeding. Currently, it is popular to identify the genetic factors controlling root system architecture by measuring root growth angles and there are evidences that the maximum root angles of primary roots are associated with shallow-rooted and deep-rooted cereal crops.
\end{abstract}

However, there are few factors affect the results of root growth angles. The growth angle of a primary root is not a constant and the maximum growth angle may not the best trait to model the root system architecture. Instead, we propose to use spatial distribution to represent the root system architecture. In order to reduce the manual involvement to save the cost and to achieve the high-throughput and accurate root phenotyping, we develop an automated image processing software solution. In this paper, we will describe the image processing solution including automatic segmentation, non-root removal, automatic detection of top root sources, the computation of the spatial distribution of roots as well as automatic counting of root tips and the measurement of total root length.

Keywords: Root phenotyping, spatial distribution, root growth angle, cereal plants, high throughput 


\section{INTRODUCTION}

Plant root development, root systems and their 3D architecture (RSA) have been subjects of extensive study for many decades (Gregory et al., 1987). This interest derives from the fact that roots are critical for plant stabilization and are the principal organs responsible for the uptake of water and nutrients from the soil. It is well known that RSA is under genetic control but can be significantly influenced by environmental factors [the genotype -by- environment $(\mathrm{G} \times \mathrm{E})$ interaction] (Fitter, 2002; Masle, 2002); the phenotype corresponding to a specific genotype is environmentally dependent (Crossa, 2012; El-Soda et al., 2014). Specific studies have documented the effects of abiotic stress on plant growth and adaptation and identified quantitative trait loci (QTLs) associated with important root traits (Kamoshita et al., 2008). With this achieved, the screening of large mapping populations then calls for highthroughput analysis methods to deal with the large number of samples possible through root plasticity (Ardiel et al., 2002; Kamoshita et al., 2008). Specifically, an important, if not indispensable, component in any high-throughput phenotyping pipeline is a robust, accurate and fully automated image analysis tool.

Despite the importance to plant phenotyping (Gerlai, 2002; Niklas and Enquist, 2002; Furbank, 2009; Harris et al., 2010), the development of automated and high-throughput analysis tools is in its adolescence, while root-based analysis specifically is in its infancy.

The most common approach to characterize roots grown in soils is to measure root traits after root extraction and washing (Iyer et al., 2010; Pierret et al., 2013). Although excavation and soil core washing in this approach destroys the 3D topology of the root system, it has been extensively used in phenotypic analyses. Indeed, even without knowledge of 3D architecture, it provides significant information on root numbers, root length, and root volume (Dowdy et al., 1998; Pierret et al., 2013; Kumar et al., 2014). Although the preparation procedure itself (root extraction, washing, cutting, and spreading on a flatbed scanner) is a tedious bottleneck for high-throughput phenotyping, the manual task of analyzing scans is an additional bottleneck. Moreover, manual analysis is the component that is most prone to subjective errors.

Image analysis techniques have been widely adopted for fast and reliable root phenotyping and made available through commercial software solutions such as WinRHIZOTM and open source software such as EZ-Rhizo (Armengaud et al., 2009), IJ_Rhizo (Pierret et al., 2013), SmartRoot (Lobet et al., 2011), RootTrace (Clark et al., 2013), RootNav (Pound et al., 2013), and Root System Analyzer (Leitner et al., 2014). With the assistance of image analysis it becomes possible to determine quantitative features such as root numbers, root diameters, root lengths as well as diameter and length distributions. These semi and fully automated software solutions were designed to analyse roots of seedlings displayed in high quality 2D scans.

For the specific purpose of $2 \mathrm{D}$ root image analysis, there are many methods developed to analyse roots (Cai et al., 2015). It is clear that a number of these can provide estimates of useful root characteristics from images in a semi-automatic way. However, there appears to be no tool that can also separate information about primary roots from that of lateral roots in a fully automatic way. Only a few of the available tools are designed to estimate the root (tip) number per primary root or per plant, which is one of the most important traits for root phenotyping (Armengaud et al., 2009). Of these, ARIA (Pace et al., 2014) requires the least user involvement. Furthermore, most use a skeletonization method to generate root representation and to count root tips by counting endpoints of the root skeleton. Regrettably, the traditional skeletonization method is sensitive to noise, thus these methods are not optimized for applications to soil-grown plants, whose scanned images may contain a significant number of soil particles contributing to noise. In summary, there is scope for further improvement.

Recently, plant biologists study root traits using a root box-pinboard method (Kano-Nakata et al., 2011). Unlike other root core washing methods, which destroy the root system architecture, this method can maintain the root system architecture in $2 \mathrm{D}$ space. Therefore, it is possible to using this approach to study the spatial distributions of plant roots as well as to study other root traits in flatbed scanning methods. As a result, we can develop algorithms for automatic counting of root tip, the measurement of total root length and the computation of the spatial distribution of roots.

In this paper, we will focus on the spatial distribution of plant roots using the image processing solution including automatic segmentation, non-root removal, automatic detection of top root sources, and the automatic computation of the spatial distribution of roots. 


\section{THE IMAGE PRE-PROCESSING FOR ROOT PHENOTYPING}

In our experiments, we used the root-box and pinboard method (Kono et al., 1987) to get images of plant roots as shown in Figure 1. Clearly, there are some issues in automatic root phenotyping using image processing, such as inhomogeneous background, pinholes in images and the automatic detection of root sources. Moreover, how to measure the spatial distribution is problematical.

\subsection{Image segmentation}

As a working principle, software tools should be designed with the ability to analyse root images obtained using different imaging tools such as flatbed scanners, and with these possibly set at different resolutions. Optimally, software solutions should thus be able to automatically adapt to variations in root images. The method employed here for segmenting plant root images has been described in detailed by Cai and Miklavcic (2013). The aim of segmentation is to separate foreground (plant roots) from background in such a way that the boundary between background and foreground can be determined easily by an edge detection method. Alternately, the edge detection method can be used to detect the boundary between foreground and background as well as to classify background points and foreground points. However, while all boundary points are edge points not all edge points are boundary points. To resolve the ensuing problem, surface fitting is used with the RANSAC

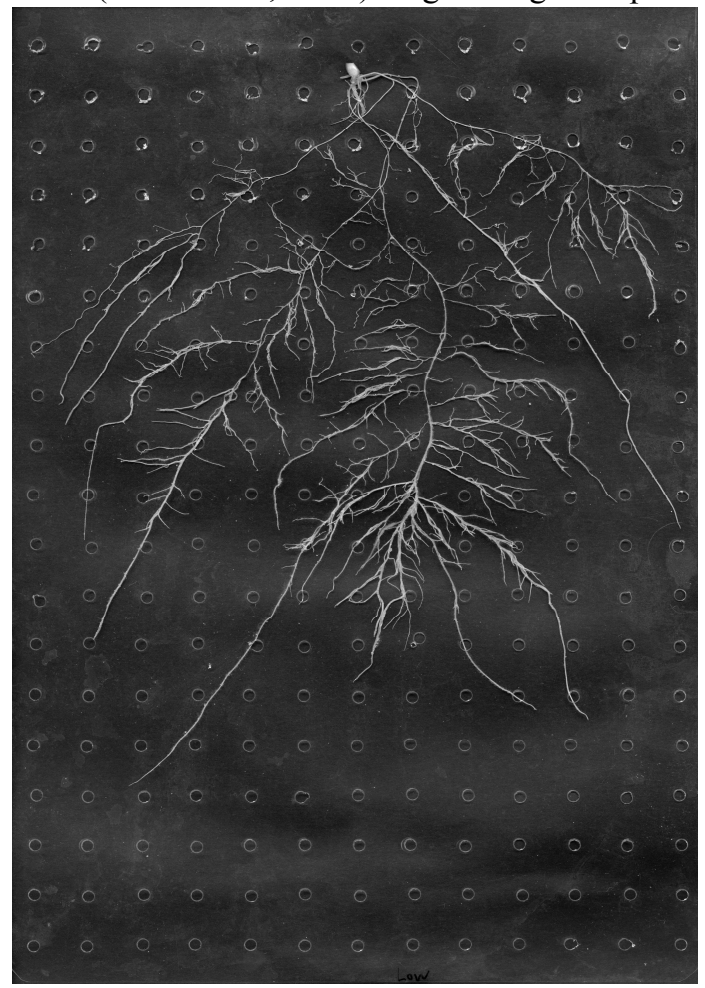

Figure 1. An example of images of plant roots from the rootbox-pinboard method. algorithm to produce an accurate estimate of surfaces and thus segment plant roots from background. The major advantage of this approach is that the background of a plant root image does not have to be homogeneous and no manual threshold is needed. An example of the segmentation results is given in Figure 2(a).

\subsection{The removal of pinholes}

It is expected that pinholes will be segmented into the foreground as pinholes have relatively sharp edges and therefore edge detection will detect them. As a result, the regions with higher intensity will be classified as foreground. As pinholes are circles, their locations and sizes can be estimated automatically using Hough transform. Based on the locations and sizes of these holes, we can remove them and then we use noise removal to eliminate the reminders. The result after the removal of pinholes is illustrated in Figure 2(b).

\section{THE SPATIAL DISTRIBUTION OF PLANT ROOTS}

\subsection{The spatial distribution of plant roots}

Usually, plant biologists use the maximum growth angle of primary roots in a plant to measure how roots of the plant spread in soil. However, the angle of the root changes due to many factors. In this paper, we will propose a novel approach to the phenotyping of root system architecture. We propose to define the spatial distribution in polar coordinate system, where its coordinates are the angle and the distance from the root source for a given root image as demonstrated in Figure 3 (a). In the current version, we set the resolution of angle as $5^{\circ}$ and the resolution of distance as $0.5 \mathrm{~cm}$. The users can also set up their preferred resolutions. Now, we need to locate the origin point by searching for the root source point. As the root at the joint of the root system and the shoot is much thicker than other root parts, it can be located by repeating the eroding operation for $\mathrm{T} / 2$ times, where $\mathrm{T}$ is the average thickness of all roots in the image. Now, most lateral roots are removed from the image, and we can find the root source (origin point) by locating the top region in the image. Now, we can represent the spatial distribution of roots in the domain of $(r, \theta)$, where $r$ is the distance from the origin and $\theta$ is the angle. 


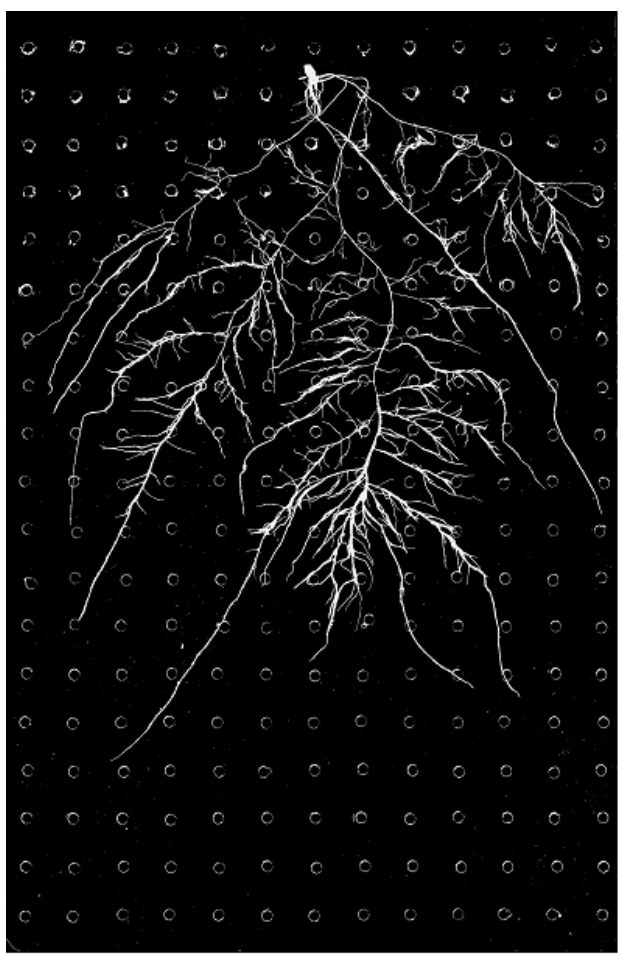

(a)

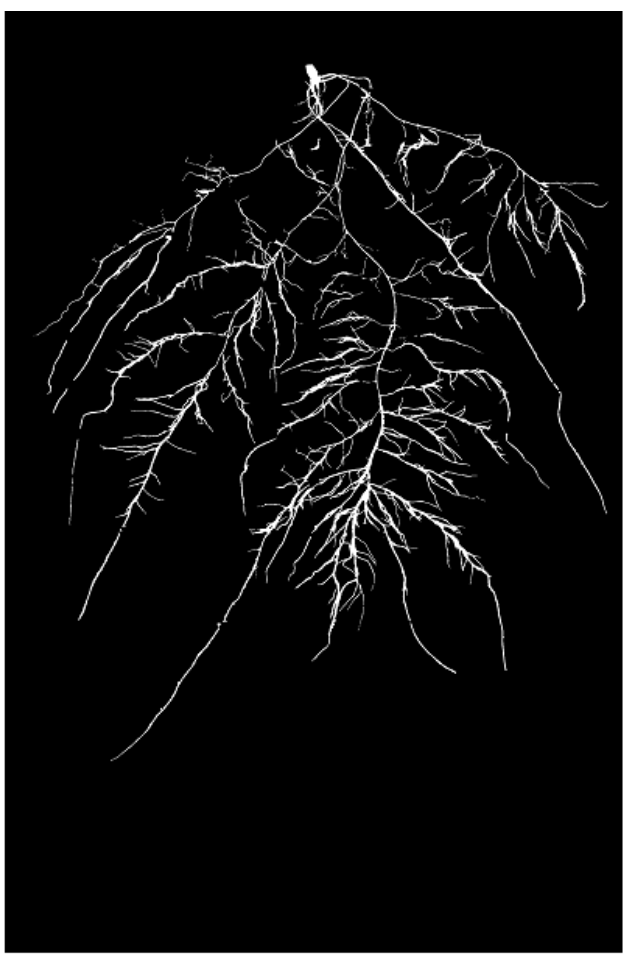

(b)

Figure 2. Root image segmentation and pinhole removal: (a) segmented image; and (b) image after pinhole removal.

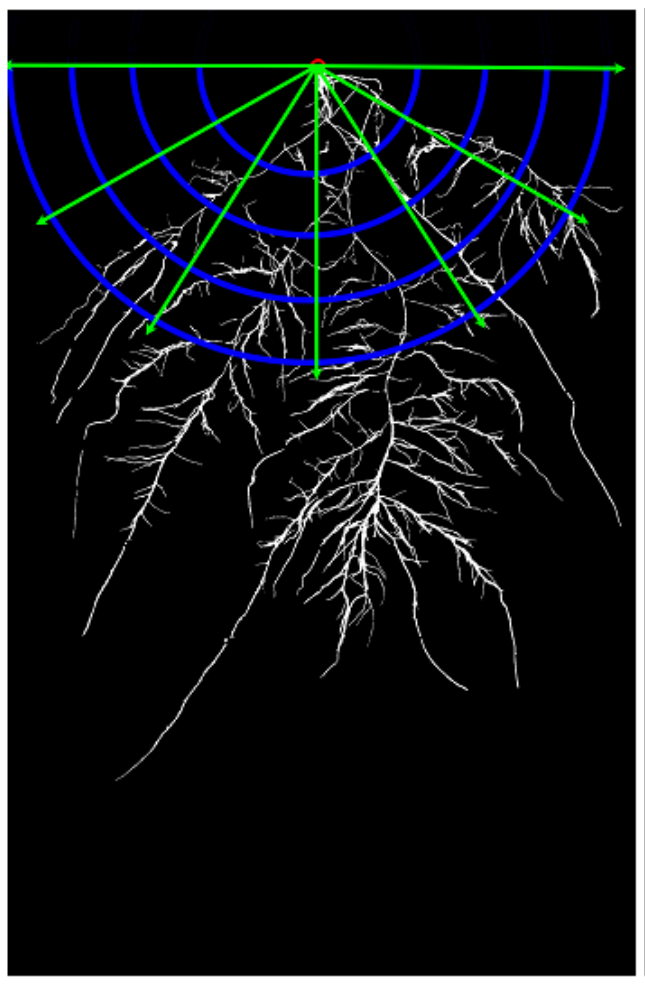

(a)

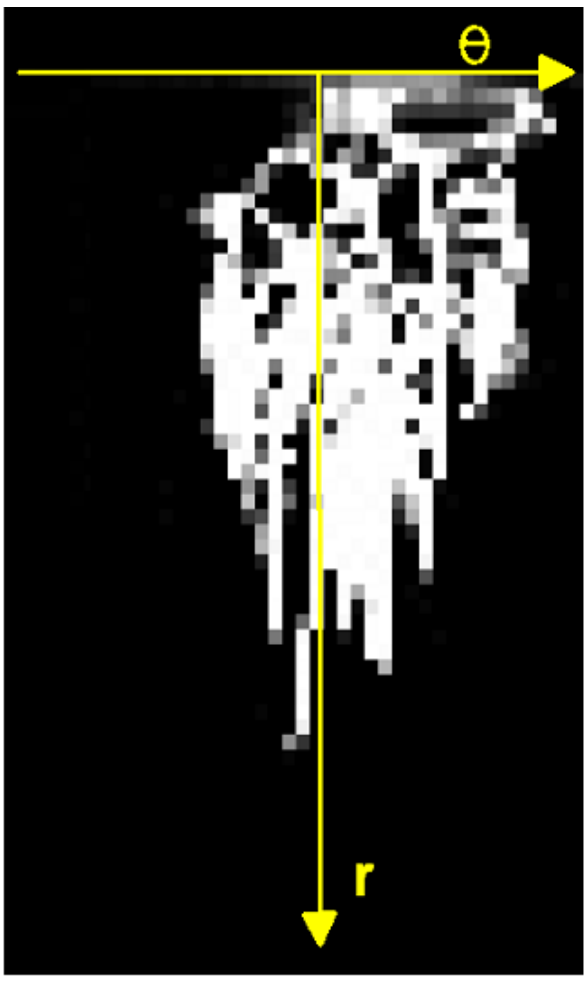

(b)

Figure 3. Root spatial distribution: (a) the polar coordinate system; and (b) the spatial distribution of the root architecture system. 


\subsection{The interpretation of the spatial distributions of plant roots}

We can use this spatial distribution to estimate many traits of the root architecture system. For the root growth angles, we can measure them at the given distance. As the distribution of roots in soil is not always evenly, the root growth angles at both sides may be useful for phenotyping shallow-rooted and deep-rooted plants. It is expected that shallow-rooted plants have more roots with small distance $r$ and less roots in deep soil, therefore, we can characterize this root trait by the ratio of roots above and under a given distance. In this case, we can avoid the problem of large variations of the maximum root growth angles of plants from the same genotypes due to the soil conditions and other factors. Many plant biologists are also interested in particular depth soil bands, and this method offers a feasible way to measure some root traits, which other approaches have difficulties to do so.

\section{DISCUSSION AND CONCLUSIONS}

Abstract: In this paper, we propose a novel approach to characterize some root traits by spatial distributions of roots. We have developed a software solution to reliably segment roots from inharmonious background, remove pinholes and compute the spatial distribution. Combining with traditional methods for measuring roots traits such as root length, tip number, volume and etc., we can provide more reliable traits of plant roots for phenotyping. This approach provides novel way to measure root growth angles at any given

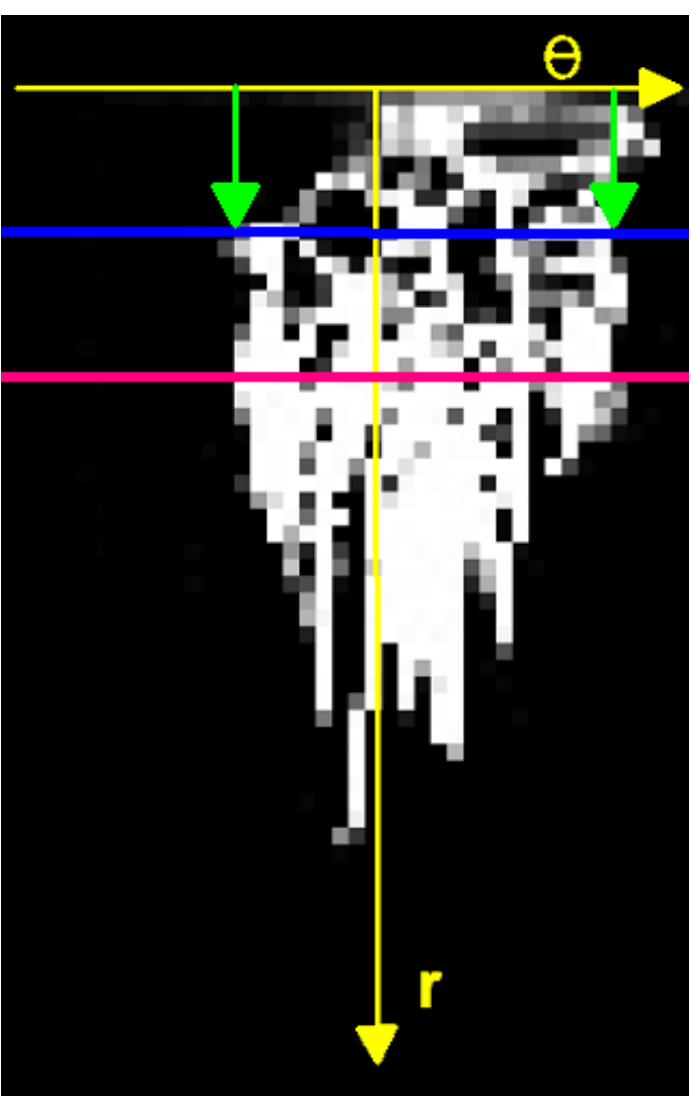

Figure 4. The interpretation of the spatial distributions.

distance. It also provides ratios of roots in soil with different depths, this is particularly useful to the phenotyping of shallow-rooted and deep-rooted crops. This approach can be applied to soil-filled glass rhizotrons for visualizing roots.

\section{ACKNOWLEDGMENTS}

The authors would like to thank Dr. Gangping Xie from CSIRO for many insightful discussions. We are grateful to the Australian Research Council, the Australian Grains Research and Development Corporation, and the South Australian Government Department of Further Education, Employment, Science and Technology for partial financial support.

\section{REFERENCES}

Ardiel G, Grewal T, Deberdt P, Rossnagel B, Scoles G. (2002). Inheritance of resistance to covered smut in barley and development of tightly linked scar marker. Theoretical and applied genetics 104, 457-464.

Armengaud P, Zambaux K, Sulpice R, Pattiso R, Blatt M, Amtmann A. (2009). EZ-RHIZO: integrated software for the fast and accurate measurement of root system architecture. The Plant Journal 57, 945956.

Cai J, Miklavcic S. (2013). Surface fitting for individual image thresholding and beyond. IET Image Processing 7, 596-605.

Cai J, Zeng Z, Connor J, Huang C, Melino V, Kumar P, Miklavcic SJ. (2015). RootGraph: a graphic optimization tool for automated image analysis of plant roots. Journal of Experimental Botany (accepted).

Clark RT, Famoso AN, Zhao K, Shaff JE, Craft EJ, Bustamante CD, Mccouch SR, Aneshansley D J Kochian LV. (2013). High-throughput two-dimensional root system phenotyping platform facilitates genetic analysis of root growth and development. Plant, cell \& environment 36, 454-466. 
Cai et al., High throughput root phenotyping

Dowdy R, Smucker A, Dolan M, Fergusson J. (1998). Automated image analyses for separating plant roots from soil debris elutrated from soil cores. Plant Soil 200, 91-94.

Fitter A. (2002). Characteristics and functions of root systems. In: Waisel Y, Eshel A, Beeckman T, Kafkafi U, eds. Plant Roots: The Hidden Half . Marcel Dekker Inc, New York, third edition, 15-32.

Furbank R. (2009). Plant phenomics: from gene to form and function. Functional Plant Biology 36, 5-6.

Gerlai R. (2002). Phenomics: fiction or the future? TRENDS in Neurosciences 25, 506-509.

Gregory P, Lake J, Rose D, eds. (1987). Root Development and Function. Cambridge University Press, Cambridge.

Harris B, Sadras V, Tester M. (2010). A water-centred framework to assess the effects of salinity on the growth and yield of wheat and barley. Plant Soil 336, 377-389.

Iyer A, Symonova O, Mileyko Y, Hao Y, Belcher H, Harer J, Weitz J, Benfey P. (2010). Imaging and analysis platform for automatic phenotyping and trait ranking of plant root systems. Plant Physiology 152 , 1148-1157.

Kamoshita A, Babu RC, Boopathi NM, Fukai S. (2008). Phenotypic and genotypic analysis of droughtresistance traits for development of rice cultivars adapted to rainfed environments. Field Crops Research $109,1-23$.

Kono Y, Yamauchi A, Nonoyama T, Tatsumi J, Kawamura N. (1987). A revised experimental system of rootsoil interaction for laboratory work. Environ. Contr. Biol. 25:141-151.

Kumar P, Huang C, Cai J, Miklavcic S. (2014). Root phenotyping by root tip detection and classification through statistical learning. Plant Soil 380, 193-209.

Leitner D, Felderer B, Vontobel P, Schnepf A. (2014). Recovering root system traits using image analysis exemplified by two-dimensional neutron radiography images of lupine. Plant Physiology 164, 24-35.

Lobet G, Pages L, Draye X. (2011). A novel image-analysis toolbox enabling quantitative analysis of root system architecture. Plant Physiology 157, 29-39.

Masle J. (2002). High soil strength: mechanical forces at play on root morphogenesis and in root: shoot signalling. In: Plant Roots: The Hidden Half. Marcel Dekker Inc, New York, third edition. 807-819.

Niklas K, Enquist B. (2002). On the vegetative biomass partitioning of seed plant leaves, stems, and roots. The American Naturalist 159, 482-497.

Pace J, Lee N, Naik HS, Ganapathysubramanian B, Lübberstedt T. (2014). Analysis of maize (zea mays 1.) seedling roots with the highthroughput image analysis tool aria (automatic root image analysis). PloS One 9, e108255.

Pierret A, Gonkhamdee S, Jourdan C, Maeght JL. (2013). IJ_RHIZO: an open-source software to measure scanned images of root samples. Plant Soil 373, 531-539.

Pound MP, French AP, Atkinson J, Wells DM, Bennett MJ, Pridmore TP. (2013). Rootnav: Navigating images of complex root architectures. Plant Physiology 162, 1802-1814. 